\title{
Erhöhtes kardiovaskuläres Risiko, aber nicht mehr Schlaganfälle
}

Fragestellung: Wie unterscheiden sich Langzeitverlauf und Komplikationen bei Diabetikern mit und ohne Vorhofflimmern?

Hintergrund: Patienten mit Vorhofflimmern haben ein erhöhtes Risiko für Schlaganfälle. Dies steigt auch bei gleichzeitigem Vorliegen eines Diabetes erheblich an. Daher geht der Diabetes in die Scores (CHAD und CHA2DS2-VASc) zur Schätzung des Schlaganfallrisikos ein, sodass bei Vorhofflimmern Diabetiker häufiger antikoaguliert werden als Nichtdiabetiker. In dieser Studie untersuchte man, wie sich die Maßnahme auf Symptome und kardiovaskuläre Kom-

\section{Originalie}

Echouffo-Tcheugui J B, Shrader $\mathrm{P}$, Thomas $\mathrm{L}$ et al. Care Patterns and Outcomes in Atrial Fibrillation Patients With and Without Diabetes (ORBIT-AF Registry). J Am Coll Cardiol 2017; 70:1325-35 plikationen auswirkt.

Patienten und Methoden: In die prospektive, US-weite Kohortenstudie gingen 9.749 Patienten (Altersmittel 75 Jahre) mit Vorhofflimmern des ORBIT-AF-Registers ein. Die 2.874 (29,5\%) Diabetiker waren im Vergleich zu den
Nichtdiabetikern jünger, hatten einen höheren Risikopunktescore für Schlaganfälle und hatten häufiger eine Hypertonie, Nierenerkrankungen, Herzinsuffizienz, eine koronare Herzerkrankung oder einen Schlaganfall. Erwartungsgemäß waren Diabetiker mit Vorhofflimmern signifikant häufiger $(p=0,001)$ antikoaguliert als Nichtdiabetiker mit Vorhofflimmern.

Ergebnisse: Diabetiker litten stärker unter den Symptomen des Flimmerns. Nach einer mittleren Beobachtungsdauer von 2,78 Jahren waren Gesamtsterblichkeit (+63\%), kardiovaskuläre Mortalität (+120\%), plötzliche Todesfälle (+53\%) und Hospitalisierungen $(+15 \%)$ bei Diabetikern mit Vorhofflimmern signifikant häufiger als bei Vorhofflimmern ohne Diabetes. Gleichzeitig waren aber thromboembolische Komplikationen wie Schlaganfall und Hospitalisierungen wegen Blutungen bei Diabetikern mit und ohne Vorhofflimmern gleich häufig.

Schlussfolgerung: Wenn Vorhofflimmern mit Diabetes einhergeht, nehmen die typischen kardiovaskulären Komplikationen zu, nicht aber die thromboembolischen Folgen und die Nebenwirkungen der Antikoagulation.

\section{- Kommentar von Prof. Dr. med. Heinrich Holzgreve}

\section{Die bittersüße Realität für Diabetespatienten}

Der Diabetes erhöht trotz aller therapeutischen Fortschritte das Risiko für kardiovaskuläre Komplikationen. Häufig ist die

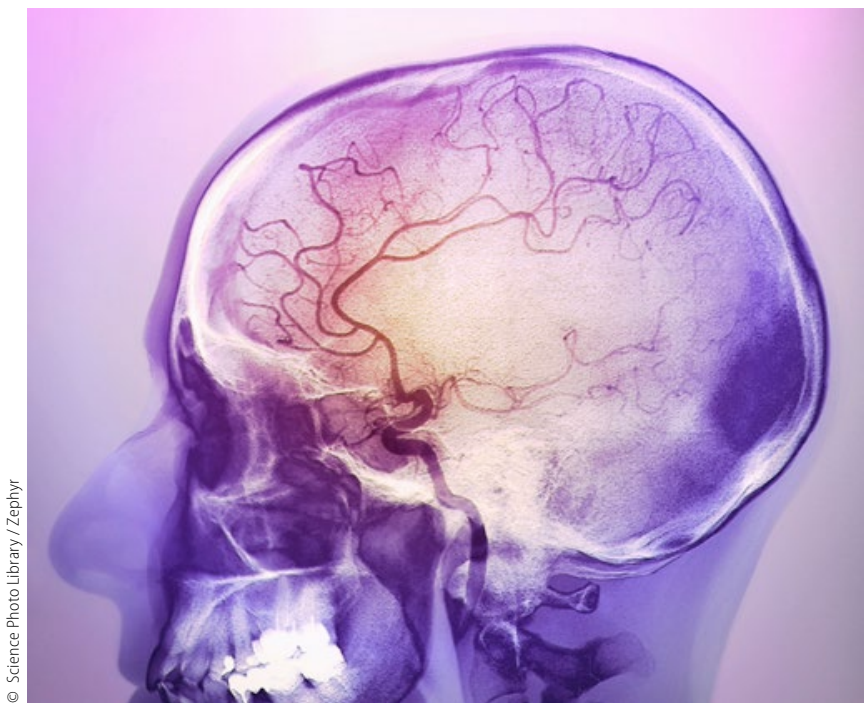

Ereignisse wie diesen Ausfall der Arteria cerebri media gilt es durch Antikoagulation bei Vorhofflimmern zu verhindern.
Erkrankung mit Vorhofflimmern assoziiert. Dann steigt das kardiovaskuläre Risiko noch weiter an. Diese bittere Wahrheit bestätigt auch die vorliegende Studie. Allerdings ergibt sich auch ein positiver Aspekt. Bei der Kombination von Diabetes und Vorhofflimmern rechnet man auch mit einem Anstieg der thromboembolischen Komplikationen, vor allem der Schlaganfälle. Deshalb geht der Diabetes in die Berechnung des Schlaganfallrisikos ein, erhöht den Score und führt häufiger zu einer Antikoagulation als bei alleinigem Vorhofflimmern.

Hier zeigt sich der süße Aspekt: Der eigentlich zu erwartende Anstieg der thromboembolischen Komplikationen kann dadurch verhindert werden.

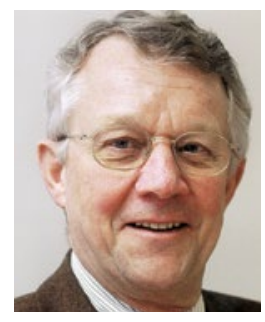

Prof. Dr. med. Heinrich Holzgreve

Internist, Kardiologische Praxis Burgstr. 7, 80331 München heinrich.holzgreve@t-online.de 